\title{
A Novel Axis HIF1A/miR-1271-5p/IncRNA PTTG3P/YAP1 Contributes to Colorectal Cancer Progression and Relates to an Immune-suppressing Microenvironment
}

\section{yang zheng}

Liaoning Cancer Institute and Hospital

ke zhang

Liaoning Cancer Institute and Hospital

yiyang liu

Youjiang Medical University for Nationalities

longfei xie

University of California Berkeley

jinnian ge

Shenyang Medical College

yue wang

Liaoning Cancer Institute and Hospital

guohua zhao ( $\nabla$ glimmerbar@163.com )

Liaoning Cancer Institute and Hospital https://orcid.org/0000-0002-0321-1925

\section{Research}

Keywords: Colorectal carcinoma, pseudogene, PTTG3P, miR-1271-5p, YAP1, HIF1A

Posted Date: April 20th, 2021

DOI: https://doi.org/10.21203/rs.3.rs-327277/v2

License: (c) (i) This work is licensed under a Creative Commons Attribution 4.0 International License. Read Full License 


\section{Abstract}

Background Pseudogene has emerged as key regulators of important biological processes involved in human cancers. However, the PTTG3P biological function in colorectal cancer (CRC) needs further to be clarified.

Methods qRT-PCR was adopted to measure the PTTG3P expression in different cell line and CRC tissues. Cellular localization of PTTG3P was detected by subcellular fractionation assay. In vitro and in vivo experiments were carried out to explore the bioeffect of PTTG3P in CRC cells. Chromatin immunoprecipitation (ChIP), luciferase assay and RIP were explored to certify the direct binding of PTTG3P and microRNA.

Results PTTG3P was upregulated in CRC and closely related to poor prognosis. Through gain and loss of function approaches, PTTG3P facilitated proliferation and glycolysis through YAP1, and glycolysis inhibitor (2-DG,3-BG) and LDHA knockdown could rescue cell proliferation and tumorigenesis. Mechanically, miR-1271-5p modulates PTTG3P expression and miR-1271-5p mimics could recover the PTTG3P function. Also HIF1A increased PTTG3P expression in both normoxia and hypoxia conditions by ameliorating miR-1271-5p expression. In addition, silencing PTTG3P decreases the level of inflammatory cytokines TNF-a, IL-1 $\beta$ and IL-6, and low PTTG3P expression relates with CD8+ T, NK and TFH cells infiltration.

Conclusions Our findings verified the oncogenic function of PTTG3P in assisting the cell proliferation and glycolysis, demonstrating the pivotal roles of HIF1A/miR-1271-5p/IncRNA PTTG3P/YAP1 in CRC progression, which proposes a novel approach for clinical treatment.

\section{Background}

Colorectal cancer (CRC) remains one of the major triggers of deaths from malignant tumors. Globally more than 1 million people suffer CRC every year[1]. As of 2012, CRC is the fourth cause of cancer death after lung, stomach, and liver cancer[2]. Despite improvements in diagnosis and combined treatment, patients with CRC have an even worse prognosis, especially in advanced patients. Therefore, it is quite urgent to clarify the mechanism, even potential approaches for the therapeutic intervention of CRC.

Accumulating evidence has shown that pseudogene, a type of long noncoding RNA, exhibits pivotal functions. It is estimated that human genome has more than 18,000 pseudogenes. And pseudogene has emerged as key regulators of important biological processes involved in the development of human cancers. For instance, increased CYP4Z1 expression promotes tumor angiogenesis and growth in breast cancer partly via PI3K/Akt and ERK1/2 activation[3]. Nanog regulates primitive hematopoiesis by directly repressing critical erythroid lineage specifiers[4]. PTENP1 can exert a growth suppressive role by regulating cellular levels of PTEN[5]. LncRNA PTTG3P (pituitary tumor-transforming 3, pseudogene, NR_002734), located at chromosome 8q13.1, was first reported in the study of human pituitary tumor 
transforming gene (hPTTG) family in 2000[6]. However, its biological function of the Warburg effect has yet to be illustrated in CRC.

During cancer progression, tumor cells acquire comprehensive metabolic reprogramming, and tissue hypoxia is a prominent feature of solid tumors leading to cell metabolism adaptive changes. miRNAs are small single-stranded non-coding RNA molecules, containing about 22 nucleotides. The function of miRNAs appears to be in gene regulation. Animal miRNAs are usually complementary to a site in the $3^{\prime}$ UTR. In our study, we identified pseudogene PTTG3P highly expressed, regulated by miR-1271-5p and hypoxia, predicted poor prognosis in patients with CRC. Further study revealed that PTTG3P could facilitate cell growth and glycolysis by regulating HIF1A/miR-1271-5p/PTTG3P/YAP1 axis and increase releasing the TNF- $a$, IL-1 $\beta$ and IL-6, because of suppressing CD $8+T$, NK and TFH cells infiltration in CRC.

\section{Materials And Methods \\ Clinical samples}

120 patients with CRC were enrolled from the Affiliated Hospital of Youjiang Medical University for nationalities, the central hospital of Shenyang medical hospital and the Liaoning cancer hospital between March 2010 and November 2015. The including criteria were as follow: patients were with a definite pathological diagnosis; No patients were received with chemotherapy or radiotherapy before surgery. The tumor and paired non-tumor tissues were also collected after lesion excision with 30mins and stored in liquid nitrogen, then transferred to $-80^{\circ} \mathrm{C}$ refrigerator. And the characteristics of cases were thoroughly noted. All of the CRC patients have signed informed consent prior to utilizing the clinical resources for investigation aims. The study was approved by the Ethics Committee of Youjiang Medical University for nationalities and China medical university.

\section{Cell lines culture}

Five human CRC cell lines (HT29, SW620, HCT-8, SW480 and HCT-116,) and normal human intestinal epithelial cell lines (FHC, NCM460) were obtained from ATCC (Manassas, VA, USA). And cultured according to their instructions. All cells were cultured in an incubator according to their instructions at 37 ${ }^{\circ} \mathrm{C}$ and in a humidified atmosphere with $5 \% \mathrm{CO} 2$.

\section{Total RNA isolation, qRT-PCR and transfection}

The expression levels of RNA were calculated by the qRT-PCR system. Total RNA was extracted by TRIzol Reagent (Invitrogen), and $1 \mu \mathrm{g}$ of total RNA was reverse transcribed using the PrimeScript RT Reagent Kit (Perfect Real-Time; Takara). pcDNA3.1-PTTG3P, PTTG3P-containing lentiviral sequence vector (shPTTG3P) were purchased from GeneChem Corporation (Shanghai, China). CRC cells were transfected with plasmids in the presence of Lipofectamine 3000 (Invitrogen). After 48h of transfection, cells were 
gathered for further use in the following experiments. The gene expression quantity was calculated using the 2- $\Delta \Delta$ Ct method. MiR-miR-1271-5p mimics miR-miR-1271-5p inhibitor and related negative controls were bought from GenePharma (Shanghai, China). The oligonucleotides were transfected into CRC cells by using Lipofectamine 2000 (Invitrogen, USA) and the transfection efficiency was confirmed by qRT-PCR. The detail is in Table S1, S2.

\section{Cell proliferation assay}

Cell viability assay was carried out to analyze cell proliferation. Cell viability was estimated using CCK8 (CK04, DOJINDO, Beijing, China), on the basis of the manufacturer's instruction. Cells were seeded in 96well culture plates. After incubation for the indicated time, CCK-8 reagent $(10 \mu \mathrm{l})$ was added to each well. Cell viability was measured with a microplate reader for the absorbance at a wavelength of $450 \mathrm{~nm}$.

\section{Flow cytometry of apoptosis}

CRC cells in 6-well plates were rinsed in phosphate buffer saline (PBS), and then were trypsinized and resuspended in $100 \mu \mathrm{L}$ binding buffer added with $2.5 \mu \mathrm{L}$ of fluorescein isothiocyanate (FITC) conjugated Annexin V and $1 \mu \mathrm{L}$ of PI (Invitrogen). Fifteen minutes later, flow cytometry (BD Biosciences) was utilized for apoptotic cells.

\section{Glucose, lactate, adenosine triphosphate (ATP) levels, and extracellular acidification rate (ECAR)}

Glucose, lactate, adenosine triphosphate (ATP) levels, and extracellular acidification rate (ECAR)

The levels of glucose and lactate were calculated with a Glucose Colorimetric Assay Kit (BioVision, CA) and a Lactate Assay Kit (BioVision, CA) in line with the instructions of manufacturer. ATP level was tested using Cell Titer-Glo Luminescent Cell Viability Assay (Promega, Madison, MI). ECAR was detected using Seahorse XF 96 Extracellular Flux Analyzer (Agilent Technologies, Santa Clara, CA) according to the manufacturer's instructions.

\section{Online databases used in this study}

PTTG3P expression in CRC cell lines was assembled by Cancer Cell Line Encyclopedia (CCLE) (www.broadinsti tute.org/ccle). Prediction of CpG islands in the PTTG3P promoter region was conducted through the MethPrimer online software (http://www.urogene.org/cgi-bin/methpri mer/methprimer.cgi) and DBCAT (http://dbcat.cgm.ntu. edu.tw/). DNA methylation analysis of CRC samples was conducted using the SMART App (http://www. bioinfo-zs.com/smartapp/) that is based on TCGA Pan-Cancer cohort of UCSC Xena public data hub (https://xenabrowser.net). 


\section{Dual-luciferase assay}

Full-length sequences of PTTG3P were PCR amplified and cloned into the psiCHECK2 vector (Promega, Madison, WI, USA). After the complementary sites for miR- 1271-5p in PTTG3P were mutated, the mutanttype (MUT) reporters MUT-PTTG3P were constructed. The luciferase reporter was co-transfected with miR-1271-5p or miR-NC into HEK-293T by Lipofectamine 2000 according to the manufacturer's guidelines. The relative luciferase activity was measured with the Dual-Luciferase Reporter Assay System (Promega) and the dual-luciferase reporter assay system (Promega) was employed for the detection.

\section{Chromatin Immunoprecipitation assay (ChIP)}

The experiments were performed according to previous reports. CRC cells $(2 \times 106)$ were prepared for ChIP assay using a ChIP assay kit (Millipore) according to the manufacturer's protocol. The resulting precipitated DNA specimens were analyzed using PCR to amplify fractions of the PTTG3P promoter. The PCR products were resolved electrophoretically on a $2 \%$ agarose gel and visualized using ethidium bromide staining.

\section{RNA immunoprecipitation (RIP)}

RIP was implemented by a Magna RIP ${ }^{\text {TM }}$ RNA-Binding Protein Immunoprecipitation Kit (Millipore, Germany). PDAC cells with indicated transfection were harvested and then lysed in the lysis buffer (50 $\mathrm{mM}$ Tris- $\mathrm{HCl}, \mathrm{pH}=7.4,150 \mathrm{mM} \mathrm{NaCl}, 1 \%$ Triton-100, 0.1\% SDS, $1.5 \mathrm{mM}$ EDTA). Thereafter, PDAC cells were lysed and incubated with protein A magnetic beads. The beads were conjugated with Ago2 antibody (Abcam, Cambridge, UK) or anti-IgG (Abcam) as negative control at $4^{\circ} \mathrm{C}$ for $6 \mathrm{~h}$. Then, the samples were digested applying Dnase I and Proteinase $\mathrm{K}$, followed by the isolation of immunoprecipitated RNA. Finally, immunoprecipitated RNA was subjected to qRT[1]PCR analysis to demonstrate the presence of PTTG3P.

\section{MS2-RIP}

Full-length PTTG3P (PTTG3P-WT) or mutant PTTG3P (PTTG3P-Mut, mutant in miR-1271-5p binding site) was cloned into the eukaryotic expression vector pSL-MS2-12X. And then these two plasmids were separately transfected into CRC cells with MS2-GFP plasmid. After $48 \mathrm{~h}$, RIP assay was performed to detect the direct interaction between PTTG3P and the miR-1271-5p. IgG and GFP antibodies (Abcam, UK) were used along with the Magna RIP RNA-Binding Protein Immunoprecipitation Kit (MilliporeSigma, Germany).

\section{Animal study}


HCT-116 cells were transfected with sh-PTTG3P. $1 \times 107$ indicated cells were subcutaneously injected into 4-week-old male nude mice. Tumor volume measured every 5 days. After 35 days, the mice were sacrificed, and the tumor weight was measured. The animal study was carried out in accordance with the Guide for the Care and Use of Laboratory Animals of the NIH. This study had been approved by the Committee on the Ethics of Animal Experiments of Youjiang Medical University for nationalities and China medical university.

\section{Statistical analysis}

All the data were shown as the mean \pm standard deviation, at least three independent experiments.

The difference between two independent groups was analyzed by a two-tailed Student's t test, while multigroup comparison was made by ANOVA. Expression correlation between genes was analyzed by Pearson correlation analysis. Survival analysis was conducted using the Kaplan-Meier method and analyzed by the log-rank test. SPSS 22.0 (SPSS Inc., Chicago, IL, USA) was used to conduct statistical analyses, and differences were ensured when P-value was $<0.05$.

\section{Results}

\section{PTTG3P is highly expressed in CRC}

To evaluate potential IncRNAs involved in mediating CRC progression, we examined the IncRNA expression profile (GSE 84983) (Fig. S1a). Comparison between CRC tumor tissues and adjacent normal tissues, we focused on the upregulated IncRNAs (fold change $>5, P<0.01$ ), for these IncRNAs might be oncogenes and therapeutic targets. LncRNA PTTG3P was one of the most upregulated and chosen for consideration (Fig. S1b). Then, we found that PTTG3P had rarely the ability to code proteins, using the open-reading frames (ORFs) Finder and conserved domain database. Moreover, five other different online metrics got the same conclusion (Table S3). Additionally, we identified no valid Kozak consensus sequence in PTTG3P[7], indicating that PTTG3P was a long noncoding RNA with no protein-coding potential. Then we explored the subcellular location of PTTG3P by using IncRNA subcellular localization predictor software (IncLocator, http://www.csbio.sjtu.edu.cn/bioinf/IncLocator/) (Fig.S1c), suggesting PTTG3P was mainly localized to the cytoplasm, and subcellular fractionation confirmed the prediction (Fig. S1d). To verify the elevation of PTTG3P in CRC, we investigated the detailed annotative process of preclinical human cancer models via the Cancer Cell Line Encyclopedia (CCLE)

(www.broadinstitute.org/ccle), indicating that PTTG3P was remarkably overexpressed in cell lines of CRC (Fig. 1a, 1b). Then, the cell lines of HT-29, SW620, HCT-8, SW480, HCT116, NCM460, and FHC were conducted for PTTG3P expression. As showed in Fig. 1c, the PTTG3P expression was exceedingly increased in HT-29, SW620, HCT-8, SW480, HCT116 cells, compared with NCM460 and FHC cells.

Further, we explored PTTG3P expression in a cohort of 120 paired and non-tumor tissues of CRC, the clinicopathologic characteristics are demonstrated in Table 1. Significantly, the PTTG3P level was 
overexpressed in CRC tissues compared to their counterparts (Fig. 1d, 1e), which was in accordance with the results of the TCGA database (Fig. 1f, 1g). Besides, high PTTG3P expression was observed in other malignant tumors (Fig. 1h). Also, our specimens confirmed PTTG3P overexpression in stomach adenocarcinoma (STAD), and esophageal squamous cell carcinoma (ESCA) (Fig. 1i,1j). Intriguingly, there was no actionable EGFR, VEGFR or RAS mutations, indicating that higher expressed PTTG3P may be driven by oncogenic event (Fig. S1e-1g). Altogether, these data revealed that PTTG3P was elevated in $\mathrm{CRC}$ and might be an oncogene.

Table 1

Correlation between PTTG3P expression and clinicopathologic characteristics of ovarian cancer patients 


\begin{tabular}{|c|c|c|c|c|}
\hline \multirow[t]{2}{*}{ Variable } & \multicolumn{3}{|c|}{ PTTG3P expression } & \multirow[t]{2}{*}{ P-value } \\
\hline & Total $(n=120)$ & High expression & Low expression & \\
\hline \multicolumn{5}{|c|}{ Age (years) } \\
\hline$\leq 60$ & 52 & 27 & 26 & \\
\hline$>60$ & 68 & 32 & 35 & 0.86 \\
\hline \multicolumn{5}{|l|}{ Gender } \\
\hline Male & 56 & 30 & 28 & \\
\hline Female & 64 & 29 & 33 & 0.74 \\
\hline \multicolumn{5}{|c|}{ Tumor size (cm) } \\
\hline$\leq 5$ & 81 & 47 & 37 & \\
\hline$>5$ & 39 & 16 & 24 & 0.02 \\
\hline \multicolumn{5}{|c|}{ Tumor invasion depth } \\
\hline T1-2 & 95 & 53 & 43 & \\
\hline T3-4 & 25 & 12 & 20 & 0.28 \\
\hline \multicolumn{5}{|c|}{ Lymph node metastasis } \\
\hline No & 40 & 25 & 20 & \\
\hline N1-2 & 80 & 36 & 39 & 0.09 \\
\hline \multicolumn{5}{|c|}{ Vessel invasion } \\
\hline Yes & 65 & 49 & 20 & \\
\hline No & 55 & 20 & 31 & 0.06 \\
\hline \multicolumn{5}{|c|}{ Differentiation } \\
\hline Well & 38 & 20 & 18 & \\
\hline Moderate & 62 & 46 & 16 & \\
\hline Poor & 20 & 13 & 7 & 0.01 \\
\hline
\end{tabular}

\section{High PTTG3P level correlates with poor prognosis}

To identify the connection between the level of PTTG3P and clinicopathologic features, we divided the cases into PTTG3P low-expression and high-expression groups on the basis of median expression. Upregulated PTTG3P was positive linked with Tumor size $(P=0.02)$ and Differentiation $(P=0.01)$, but not with age $(P=0.86)$, gender $(P=0.74)$, tumor invasion depth $(P=0.28)$, lymph node metastasis $(P=0.09)$ or 
vessel invasion ( $\mathrm{P}=0.06$ ) (Table 1). Moreover, the PTTG3P expression was higher in stage III-IV (advanced stage) than stage I-II (early stage) in tissues (Figure 2a). Additionally, Kaplan-Meier survival curves illustrated that patients with highly expressed PTTG3P had poorer survival time (Figure 2b). Further, we determined the prognostic ability of PTTG3P in CRC. As shown in Table 2, univariate analyses suggested highly expressed PTTG3P was associated with a dramatic risk of death $(P<0.01)$. Multivariate analysis demonstrated that PTTG3P expression could be an independent prognostic factor $(P<0.01)$. A model that incorporated the independent predictor was proposed as the nomogram (Fig. S2). Subsequently, the ROC curve was carried out to evaluate the diagnostic value of PTTG3P in CRC tissues compared with normal counterparts, the area under the ROC curve (AUC) was 0.776 (95\% Cl 0.733-0.819) (Figure 2c). Thus, these data suggested that high expression of PTTG3P predicted a worse prognosis and may serve as a clinical biomarker for CRC patients.

Table 2

Univariate and multivariate analyses of clinicopathologic characteristics for correlations with overall survival

\begin{tabular}{|c|c|c|c|c|}
\hline \multirow[t]{2}{*}{ Variables } & \multicolumn{2}{|l|}{ Univariate analysis } & \multicolumn{2}{|l|}{ Multivariate analysis } \\
\hline & $\mathrm{HR}(95 \% \mathrm{Cl})$ & $P$ value & $\mathrm{HR}(95 \% \mathrm{Cl})$ & $P$ value \\
\hline PTTG3P expression & $1.758(1.085-2.850)$ & $<0.01$ & $1.712(1.053-2.782)$ & $<0.01$ \\
\hline Tumor size & $1.650(1.086-2.508)$ & $<0.01$ & $1.923(1.276-2.898)$ & $<0.01$ \\
\hline Differentiation & $1.724(1.183-2.511)$ & $<0.01$ & $1.724(1.183-2.511)$ & $<0.01$ \\
\hline
\end{tabular}

\section{PTTG3P is caused by metabolic stress and promotes glycolysis and proliferation in CRC}

To investigate the biological function of PTTG3P, we transfected the PTTG3P overexpressed plasmids and shRNA targeting PTTG3P into HT-29 and HCT116 cells, respectively (Fig. 2d). By determining PTTG3P expression via gene set enrichment analysis (GSEA) the Cancer Genome Atlas (TCGA) profiles, we found that PTTG3P level was positively correlated with the glycolysis by affecting genes in glycolysis regulation (Fig. 2e). PTTG3P knockdown restrained the mRNA level of GLUT-1, ALDOA, PKM2, and LDHA, and the effect of sh-PTTG3P on glycolytic gene transcription could be rescued by PTTG3P re-expression (Fig. 2f). And glucose deprivation is a well-known feature of solid tumors. Subsequently, we wonder whether PTTG3P participated in cell survival under metabolic stress, then we carried out several experiments with different glucose concentrations and glycolysis inhibitor 2-deoxyglucose (2-DG) to make a condition of glucose deprivation. Obviously, PTTG3P expression was increased by glucose 
deprivation or 2-DG treatment in either dose-dependent or time-dependent manner (Fig. 2g, 2h). Thus, we elucidated that PTTG3P could play a crucial role in the progression of metabolic stress.

Next, we performed the glucose uptake analysis, ATP analysis, lactate production analysis, and discovered that sh-PTTG3P repressed these phenomena. In contrast, PTTG3P overexpression boosted glucose uptake (Fig. 3a), lactate production (Fig. 3b), and ATP accumulation (Fig. 3c). Additionally, we calculated the level of ECAR, sh-PTTG3P notably repressed glycolytic capacity and vice verse (Fig. 3d). Also, we found that silenced PTTG3P suppressed the proliferation, facilitated apoptosis of HCT116 cells, whereas upregulated PTTG3P increased the proliferation, inhibited apoptosis of HT-29 cells according to the CCK-8 assay and flow cytometry analysis (Fig. 3e,3f). In vivo, highly expressed PTTG3P efficiently increased the tumor growth (Fig. 3g,3h). We then explored whether glycolysis played a vital role in cell proliferation and tumor growth. Notably, the glycolic inhibitors 2-DG and 3-BP or depletion of LDHA, which catalyzed the final step of glycolysis, could partly abrogate cancer cell proliferation and tumor growth (Fig. 3i,3j,3k). Clinically, Oxaliplatin is used for the treatment of colorectal cancer. Previously, it is reported that suppression of glycolysis is an effective strategy to block cell proliferation and conquer drug resistance. As shown in Fig. 31,3m, PTTG3P depletion and Oxaliplatin played a synergistic role in emancipating tumor growth. As a taken, PTTG3P knockdown plus Oxaliplatin is a promising therapy for CRC.

\section{PTTG3P regulates Hippo signaling pathway in CRC}

In order to elucidate which pathway is involved in PTTG3P-mediated CRC progression, GSEA in the published TCGA CRC database was explored. And we suggested that PTTG3P expression was associated with the YAP1-activated gene signatures, indicating that Hippo signaling pathway might be involved (Fig. 4a). Then the hub genes in the Hippo pathway, including LATS1/2, MST1/2 and YAP1, and Hippo pathway target genes, such as CDX2, FOXM1, CTGF and CYR61, were checked in sh-PTTG3P HCT116 cells. Subsequently, PTTG3P knockdown impaired the level of YAP1, FOXM1 and CTGF (Fig. 4b). Moreover, silenced PTTG3P decreased the enrichment of H3K27Ac at the YAP1 promoter, while that of H3K27me3 was increased (Fig. 4c). Next, we explored the TCGA database and drew the PTTG3P coexpression heat map. Then, we discovered that PTTG3P expression was correlated with genes in the Hippo pathway (YAP1, TEAD1-3), genes in the phenotype of proliferation (PCNA, MKI67, MCM2, MCM3, MCM5), genes in the phenotype of apoptosis (BAX, CASP1, CASP3, CASP10), genes in the phenotype of autophagy (ATG5), and genes in the phenotype of cell cycle (CDK1, CCDN1, CCND2, CCNB1), but not with MST1, an upstream factor of YAP1 (Fig. 4d).

It is commonly acknowledged that YAP1, a crucial factor in the Hippo pathway, involves in cell proliferation and suppresses apoptotic genes, and YAP1 was highly expressed in CRC (Fig. S1h, S1i), and associated with advanced characteristics of CRC (Table S4). Further, YAP1 had a higher diagnostic value (AUC=0.793, 95\% Cl:0.729-0.858) from the TCGA database (Fig. S1j). Additionally, we performed rescue assays in HT-29 cells. PTTG3P OE plus YAP1 KD could reverse the bioeffect of PTTG3P. Besides, we 
applied YAP1 inhibitors, CA3 (a novel specific YAP1 inhibitor) and verteporfin (an inhibitor of YAP1/TEAD interaction), got the same conclusion (Fig. 4e-4h). Intriguingly, the treatment of Hippo pathway inhibitor, XMU-MP-1 (inhibiting MST1/2), could not recover the effect of PTTG3P on proliferation, apoptosis and tumor growth (Fig. 4i, 4j).In brief, all the data uncovered that PTTG3P hedges the key factor MST1/2, while modulates YAP1 in the Hippo pathway to exhibit pivotal functions in CRC progression.

\section{Methylation and deacetylation are not involved in PTTG3P upregulation in CRC}

We first determined whether DNA methylation can regulate PTTG3P expression. No CpG islands were found in the PTTG3P promoter, as shown by analyzing PTTG3P promoter sequences via the online software MethPrimer (http://www.urogene.org/cgi-bin/methprimer/methprimer.cgi) and DBCAT (http://dbcat.cgm. ntu.edu.tw/) (Figure 5a, b). To validate the role of DNA methylation on the regulation of PTTG3P expression, HT29 and HCT-116 cells were transfected with small interfering RNA (siRNA) for DNA (cytosine-5)-methyltransferase 1,3A,3B (DNMT1,3A,3B), crucial enzymes catalyzing the transfer of methyl groups to certain $\mathrm{CpG}$ structures of DNA. Our results demonstrated that these DNA methylation enzymes could barely influence PTTG3P expression(Figure 5c). Additionally, we treated CRC cells with 5 $\mu \mathrm{M}$ 5-azacytidine (5-AZA), an inhibitor of DNA methylation, our results revealed that 5-AZA treatment did not affect PTTG3P levels in CRC cells (Figure 5d). Accumulating evidence has shown that ectopic expression of IncRNAs could be regulated by transcriptional factors, and histone acetylation plays a critical role in this procession. Then, we carried out experiments with SAHA and NaB, the broad-spectrum HDAC inhibitors, and we discovered that these HDAC inhibitors failed to alter PTTG3P level in HT29 cells (Figure 5e). In addition, overexpressed HDAC6 and HDAC8 did not affect increasing PTTG3P expression (Figure 5f). Next, histone methylation may also influence gene transcription. We knocked down EZH2 and LSD1 in HT29 and HCT-116 cells and measure the PTTG3P expression. As a result, silencing EZH2 or LSD1 did not markedly influence PTTG3P expression, indicating that histone methylation is not involved in PTTG3P upregulation in CRC cells (Figure $5 \mathrm{~g}$ ). In summary, our findings preliminarily demonstrated that methylation and deacetylation are not participating in the upregulation of PTTG3P in CRC.

\section{miR-1271-5p regulates PTTG3P expression and rescues the function of PTTG3P}

Immunoprecipitation assay confirmed that PTTG3P bound to Ago2, the main component of the microRNA-related silencing complex (Fig. 6a). We assumed that PTTG3P could be regulated by miRNAs. MicroRNAs (miRNAs) are endogenous small non-coding RNAs, binding to the 3'-untranslated regions (3'UTRs) of target genes to regulate target gene expression. Bioinformatics analysis was performed to predict the candidate microRNAs. The most promising candidate gene is miR-1271-5p, which is relatively low expressed in CRC (figure 6b, 6c) and has a diagnostic value with an AUC of $0.919(95 \% \mathrm{Cl}, 0.861$ 0.977) (figure 6d). Ago2 RIP assay showed that miR-1271-5p was the highest enriched microRNAs in 
PTTG3P overexpression group than the vector group (Fig. 6e). PTTG3P expression was suppressed by overexpression of miR-1271-5p but promoted by the specific microRNA inhibitors (Fig. 6f, 6g). Next, we designed a reporter construct in which the putative miR-1271-5p binding sites in the PTTG3P sequence were mutated by site-directed mutagenesis (Fig. 6h). Transfection of miR-1271-5p mimics significantly inhibited the luciferase activity of reporters containing PTTG3P-WT, instead of PTTG3P-Mut (Fig. 6i). MS2-RIP assay was used to further verify the direct interaction between miR-1271-5p and PTTG3P. The MS2-tagged wild-type PTTG3P vector was enriched for miR-1271-5p compared to the empty and mutant plasmids (Fig. 6j). These data strongly suggested that PTTG3P could be regulated by miR-1271-5p in CRC cells. Besides, higher miR-1271-5p expression associated with advanced characteristics (Table S5), and poor prognosis (Fig. 6k). Intriguingly, miR-1271-5p mimics could abolish the PTTG3P function (figure $7 a-7 g)$.

We also investigated whether hypoxia could regulate PTTG3P expression in CRC cells.

Then, we treated CRC cells with hypoxia or CoCl2 (hypoxia chemical inducer) and found the PTTG3P expression in HT-29 cells was obviously increased as well as the elevation of HIF1A (Fig. 7h). While, depletion of HIF1A strikingly ameliorated PTTG3P expression in both normoxia and hypoxia conditions (Fig. 7i, 7j). We also indicated that HIF1A and PTTG3P had a positive correlation (Fig. 7k, S3h). Interestingly, HIF1A could diminish miR-1271-5p expression (Fig. 7l). Hence, HIF1A/miR-12715p/PTTG3P/YAP1 axis conducted a pivotal role in CRC progression.

\section{PTTG3P plays crucial functions in cancer immunology}

Cancer cells have high glucose uptake and glycolysis, resulting in a low level of glucose in the tumor, thus inhibiting the production of IFN-r by $\mathrm{CD} 8+\mathrm{T}$ cells in the tumor. Tumor immune environment is widely involved in tumor progression different malignant tumors, including CRC. Recently, the treatment of CRC with immune checkpoint inhibitors (ICI) has provided a potential clinical treatment. Interestingly, our findings from the TCGA database proposed that low PTTG3P expression relates with CD8+ T, NK and TFH cells infiltration in the microenvironment of CRC, not with Treg or macrophages infiltration (Figure S3a-f). And the results of ELISA showed that the level of inflammatory cytokines TNF-a, IL-1 $\beta$ and IL-6 were decreased with PTTG3P depletion (Figure S3g).

In addition, PTTG3P was correlated with TNF-a, IL-1 $\beta$ and IL-6 from the TCGA-COAD database (Fig. S3h).

\section{Discussion}

Pseudogene may be transcribed into RNA at low levels, due to promoter elements inherited from the ancestral gene or arising by new mutations. Although most transcripts have rarely functional significance than chance transcripts from other parts of the genome, some have given rise to regulatory RNAs and new proteins. For instance, long noncoding RNA HK2P1, a pseudogene of HK2, promoted lactate production and glucose uptake in endometrial stromal cells[8]. Pseudogene PTENP1 repressed the 
oncogenic PI3K/AKT pathway and inhibited the HCC progression[9]. To date, the role of pseudogene PTTG3P in glycolysis of CRC has not been reported yet. Our findings documented that PTTG3P facilitated CRC progression via the HIF1A/miR-1271-5p/PTTG3P/YAP1 axis.

Our study verified that PTTG3P is highly expressed and has a potential diagnostic value, with an AUC of 0.776 (95\% Cl 0.733-0.819) in CRC. Clinically, high PTTG3P expression considerably associates with tumor size and TNM stage as well as shorter survival time. These results confirmed that PTTG3P serves as a valuable prognostic biomarker and aids innovatively efficient therapies for CRC patients. Additionally, our findings stand in line with other research, Liu, et al.[10] reported that PTTG3P was remarkably upregulated in CRC tumor samples than that in normal samples. Zhou, et al.[11] revealed that PTTG3P is a valuable resource for identification in HCC progression and is useful for biomarker development. Weng, et al.[12] certified that PTTG3P facilitates cell proliferation, migration and invasion and might serve as a new promising strategy for gastric cancer. Recently, PTTG3P expression has a relationship with breast cancer[13] and pancreatic cancer[14]. Thus, the oncogenic role of PTTG3P in malignant tumors is strongly suggested.

Malignant tumors could undergo glycolysis at a higher speed than that of non-tumor tissue controls[1517]. This phenomenon is known as the Warburg effect[18]. The Warburg hypothesis demonstrates that malignant tumor is fundamentally caused by mitochondrial metabolism disorder. Doherty JR, et al.[17] found that tumor lactate levels correlate with increased metastasis, tumor recurrence, and poor outcome. And targeting lactate metabolism is a prospective method for cancer therapeutics. Furthermore, cancer cells with high level of glycolysis and acid resistance have an energetic growth advantage, which facilitates unrestrained proliferation and invasion. In our study, we explored gain and loss-of-function approaches in HT29 and HCT-116 cells and found PTTG3P ablation resulted in the inhibition of CRC cell glycolysis by regulating numerous genes linked with metabolic pathways, whereas the opposite outcome was observed after enforced expression of PTTG3P. Nowadays, ketogenic diet was used to constrain glycolysis to starve cancer cells, adjusting mitochondrial metabolism[19]. Here, we also proposed that the biological mechanism of PTTG3P on boosting cell proliferation might resist apoptosis.

Hippo signaling pathway has become increasingly important in human cancer[20], the key regulator YAP1 has been certified to be upregulated in breast cancer, colorectal cancer, and liver cancer[21], and YAP1 could promote cell growth[22-24] and inhibit apoptosis[25]. Clinically, YAP1 could be a target for the development of cancer drugs[26]. Yi, et al. [27] suggested that inhibiting TEAD-YAP1 interactions or block the binding function of WW domains is a pharmacologically viable strategy against the YAP1 oncoprotein. In our presented study, we discovered that PTTG3P activates Hippo signaling pathway by promoting YPA1, FOXM1 and CTGF, not MST1/2, and rescue assay consolidate this by using the Hippo pathway inhibitor, XMU-MP-1 (inhibiting MST1/2).

Many other miRNAs also have links with cancer and accordingly are sometimes referred to as "oncomirs". Another role for miRNA in cancers is to use their expression level for prognosis. In NSCLC samples, low miR-324a levels may serve as an indicator of poor survival[28]. Either high miR-185 or low miR-133b 
levels may correlate with metastasis and poor survival in colorectal cancer[29]. Furthermore, specific miRNAs may be associated with certain histological subtypes of colorectal cancer. For instance, expression levels of miR-205 and miR-373 are increased in mucinous colorectal cancers and mucinproducing Ulcerative Colitis-associated colon cancers, but not in sporadic colonic adenocarcinoma that lacks mucinous components[30]. Then, we investigated the upstream factor of PTTG3P in CRC and found miR-1271-5p could regulate the PTTG3P expression. Additionally, we carried out a series of rescue experiments to verify miR-1271-5p mimics could ablate the bioeffect of PTTG3P. Intriguingly, HIF1A increased PTTG3P expression by decreasing the miR-1271-5p level (Fig. 7m). Besides, our findings from the TCGA database proposed that low PTTG3P expression relates with CD8 + T, NK and TFH cells infiltration in the microenvironment of $\mathrm{CRC}$, not with Treg or macrophages infiltration. And we found that a decreased TNF-a, IL-1 $\beta$ and IL-6 level was accompanied by ablation of PTTG3P.

\section{Conclusion}

In conclusion, our data proposed that PTTG3P is upregulated in CRC cell lines and tissues, and is related to poorly prognosis in CRC patients. HIF1A/miR-1271-5p/PTTG3P/YAP1 axis plays a vital part in CRC progression. Clinically, PTTG3P could be a potential therapeutic target.

\section{Declarations}

\section{Ethics approval and consent to participate}

Patients have given their written informed consent in our study. And ethics committee of Cancer Hospital of China Medical University an.d Youjiang Medical University for Nationalities

\section{Consent for publication}

All the authors agreed to publish the manuscript.

\section{Availability of data and material}

The datasets used and analyzed in the current study are available from the corresponding author on reasonable request.

\section{Competing interests}

The authors declare that there are no competing interests associated with the manuscript.

\section{Funding}


Supported by Natural Science Foundation of Liaoning Province of China.

\section{Authors' contributions}

The work presented here was carried out in collaboration between all authors. YW and GZ contributed to the conception of the study; $Y Z, K Z$ and $Y L$ contributed significantly to carry out the experiment. LX, JG performed the data analyses and wrote the manuscript;

\section{Acknowledgment}

We appreciate all the participants who provide supports for our research.

\section{References}

1. Cunningham D, Atkin W, Lenz HJ, et al. Colorectal cancer. Lancet.2010, 375 (9719): 1030-1047.

2. Adelstein BA, Macaskill P, Chan SF, et al. Most bowel cancer symptoms do not indicate colorectal cancer and polyps: a systematic review. BMC Gastroenterology. 2011;11:65.

3. Rieger MA, Ebner R, Bell DR, et al. Identification of a novel mammary-restricted cytochrome P450, CYP4Z1, with overexpression in breast carcinoma. Cancer Res.2004, 64, 2357-2364.

4. Wei $\mathrm{Yu} \mathrm{H}$, Chai Y, Li, et al. Increased expression of CYP4Z1 promotes tumor angiogenesis and growth in human breast cancer. Toxicol Appl Pharmacol. 2012;264(1):73-83.

5. Poliseno L, Salmena L, Zhang J, et al. A coding-independent function of gene and pseudogene mRNAs regulates tumour biology. Nature. 2010;24(7301):1033-8. 465 ).

6. Chen L, Puri R, Lefkowitz EJ, Kakar SS. Identification of the human pituitary tumor transforming gene (hPTTG) family: molecular structure, expression, and chromosomal localization. Gene. 2000;248:4150 .

7. Kozak M. Point mutations define a sequence flanking the AUG initiator codon that modulates translation by eukaryotic ribosomes. Cell. 1986;44:283-92.

8. Lv H, Tong J, Yang J, et al. Dysregulated Pseudogene HK2P1 May Contribute to Preeclampsia as a Competing Endogenous RNA for Hexokinase 2 by Impairing Decidualization. Hypertension. 2018;71(4):648-58.

9. ChiuLing Chen YenWen, Wu TJawChing, et al. Suppression of hepatocellular carcinoma by baculovirus-mediated expression of long non-coding RNA PTENP1 and MicroRNA regulation. Biomaterials. 2015;44:71-81.

10. Liu N, Dou L, Zhang X. LncRNA PTTG3P Sponge Absorbs microRNA-155-5P to Promote Metastasis of Colorectal Cancer. 2020,(9)13:5283-5291.

11. Zhou Q, Zhang W, Wang Z, et al. Long non-coding RNA PTTG3P functions as an oncogene by sponging miR-383 and up-regulating CCND1 and PARP2 in hepatocellular carcinoma. BMC Cancer. 
2019;24(1):731. 19(.

12. Weng W, Ni S, Wang Y, et al. PTTG3P promotes gastric tumour cell proliferation and invasion and is an indicator of poor prognosis. J Cell Mol Med. 2017;21(12):3360-71.

13. Lou W, Ding B, Fan W. High Expression of Pseudogene PTTG3P Indicates a Poor Prognosis in Human Breast Cancer. Mol Ther Oncolytics. 2019;27:14:15-26.

14. Liu W, Tang J, Zhang H, et al. A novel IncRNA PTTG3P/miR-132/212-3p/FoxM1 feedback loop facilitates tumorigenesis and metastasis of pancreatic cancer. Cell Death Discov. 2020;30(1):136. 6(.

15. GATENBY R A, GILLIES RJ. Why do cancers have high aerobic glycolysis? Nat Rev Cancer. 2004;4(11):891-9.

16. OU J, MIAO H, MA Y, et al. Loss of abhd5 promotes colorectal tumor development and progression by inducing aerobic glycolysis and epithelial-mesenchymal transition. Cell Rep. 2014;9(5):1798-811.

17. CLEVELAND DOHERTYJR. J L. Targeting lactate metabolism for cancer therapeutics. J Clin Invest. 2013;123(9):3685-92.

18. WARBURG O. On the origin of cancer cells. Science. 1956;123(3191):309-14.

19. SCHWARTZ L, SEYFRIED T, ALFAROUK KO, et al. Out of Warburg effect: An effective cancer treatment targeting the tumor specific metabolism and dysregulated pH. Semin Cancer Biol, 2017, 4313443138.

20. Saucedo LJ, Edgar BA. Filling out the Hippo pathway. Nature Reviews Molecular Cell Biology. 2007;8(8):613-21.

21. Zender $L$, Spector $M S$, Xue W, et al. Identification and validation of oncogenes in liver cancer using an integrative oncogenic approach.Cell. 2006,30; 125(7): 1253-1267.

22. Shimomura T, Miyamura N, Hata S, et al. The PDZ-binding motif of Yes-associated protein is required for its co-activation of TEAD-mediated CTGF transcription and oncogenic cell transforming activity. Biochem Biophys Res Commun. 2014;443(3):917-23.

23. Harvey KF, Zhang X, Thomas DM. The Hippo pathway and human cancer. Nature Reviews Cancer. 2013;13(4):246-57.

24. Johnson R, Halder G. The two faces of Hippo: targeting the Hippo pathway for regenerative medicine and cancer treatment. Nature Reviews Drug Discovery. 2014;13(1):63-79.

25. Zhao B, Kim J, Ye X, et al. Both TEAD-binding and WW domains are required for the growth stimulation and oncogenic transformation activity of yes-associated protein. Can Res. 2009;69(3):1089-98.

26. Sudol M, Shields DC, Farooq A. Structures of YAP protein domains reveal promising targets for development of new cancer drugs. Semin Cell Dev Biol. 2012;23(7):827-33.

27. Liu-Chittenden Y, Huang B, Shim JS, et al. Genetic and pharmacological disruption of the TEAD-YAP complex suppresses the oncogenic activity of YAP. Genes Dev. 2012;26(12):1300-5.

28. Michel JWeber. New human and mouse microRNA genes found by homology search.2005, 272(1):59-73. 
29. Young-Kook Kim VN, Kim. Processing of intronic microRNAs. EMBO J. 2007;7(3):775-83. 26 ).

30. Baskerville S, Bartel DP. Microarray profiling of microRNAs reveals frequent coexpression with neighboring miRNAs and host genes. RNA. 2005;11(3):241-7.

\section{Figures}

A
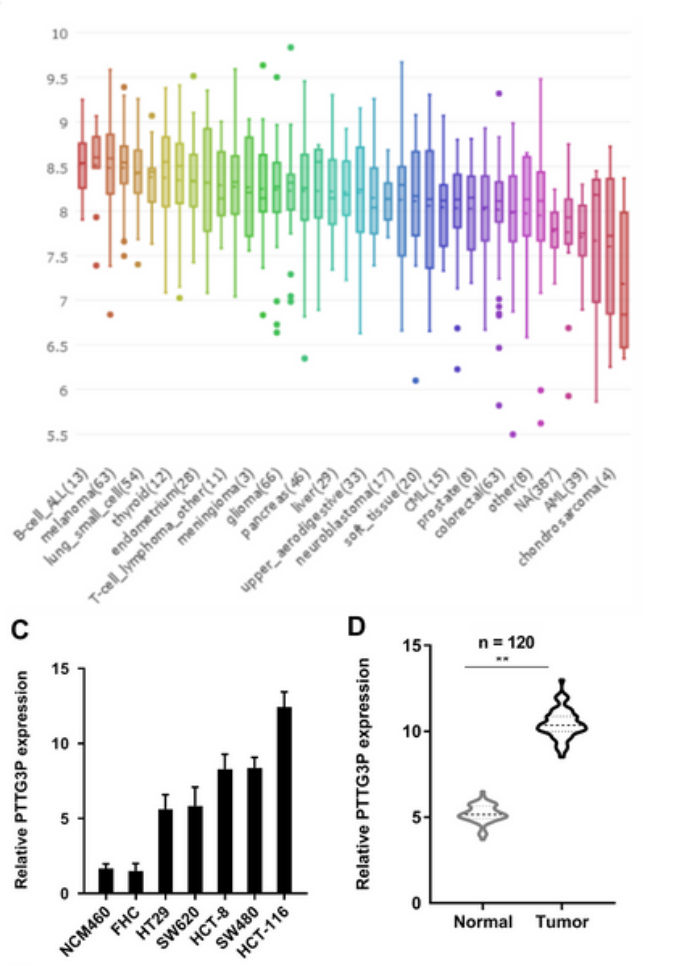

H
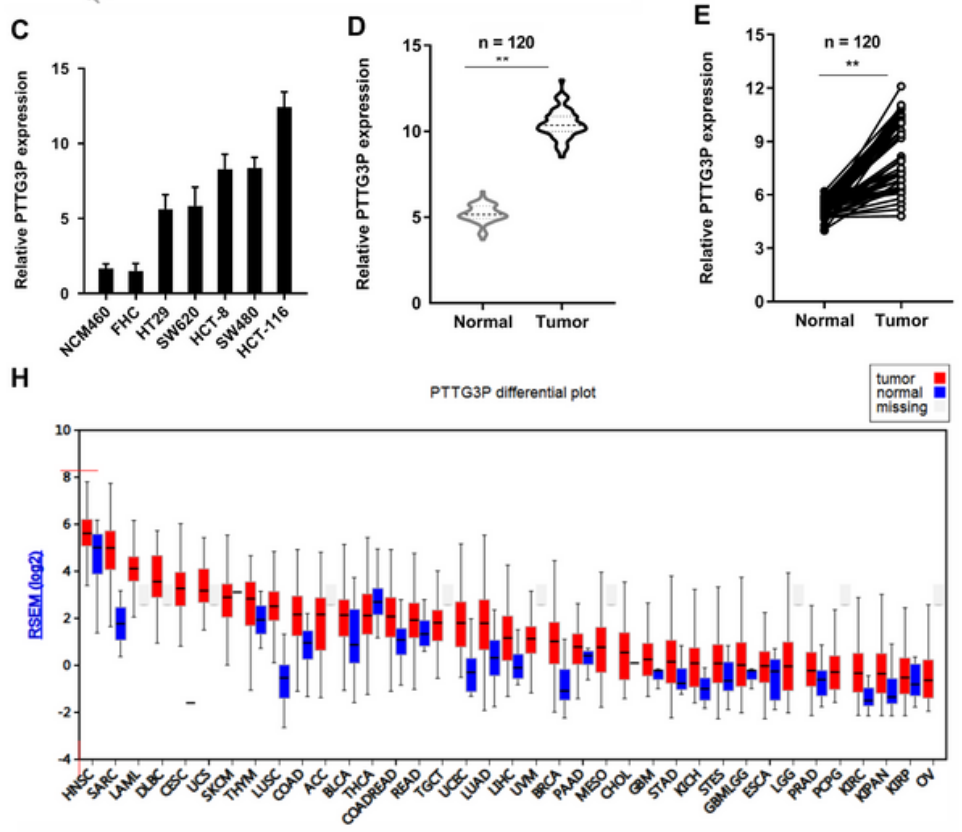

B
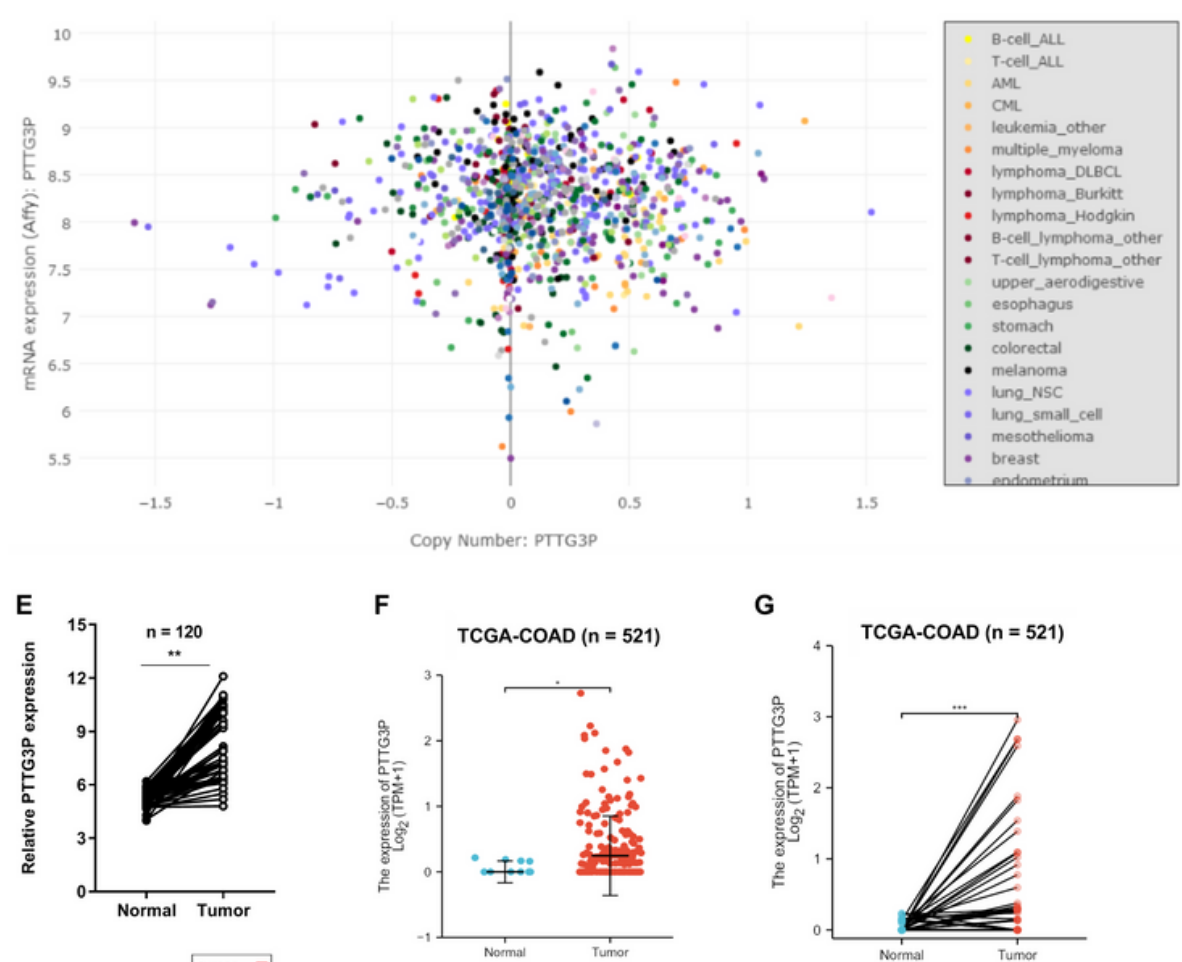

G
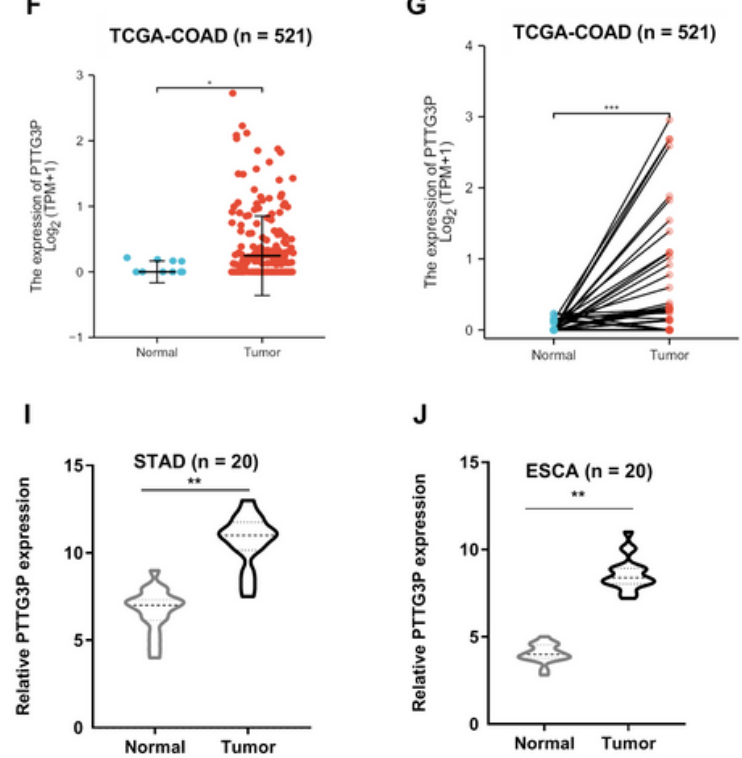

\section{Figure 1}

PTTG3P is upregulated in CRC tissues, and cell lines. (A, B) Exploring PTTG3P expression in CRC cell lines by assembling the Cancer Cell Line Encyclopedia (CCLE) (www.broadinstitute.org/ccle). (C) The expression profiles of PTTG3P in HT-29, SW620, HCT-8, SW480, HCT116, NCM460 and FHC was detected with qRT-PCR. (D, E) qRT-PCR analysis was used to evaluate PTTG3P expression in 120 paired tumor and paired adjacent non-tumor tissues. (F, G) High PTTG3P expression was observed in the TCGA database of CRC ( $n=521)$. $(H)$ High PTTG3P expression was observed in the TCGA database of malignant tumors. 
$(\mathrm{I}, \mathrm{J})$ High PTTG3P expression was observed in STAD and ESCA $(n=20) .{ }^{*}<<0.05, * \star P<0.01, * \star * P<$ 0.001

A

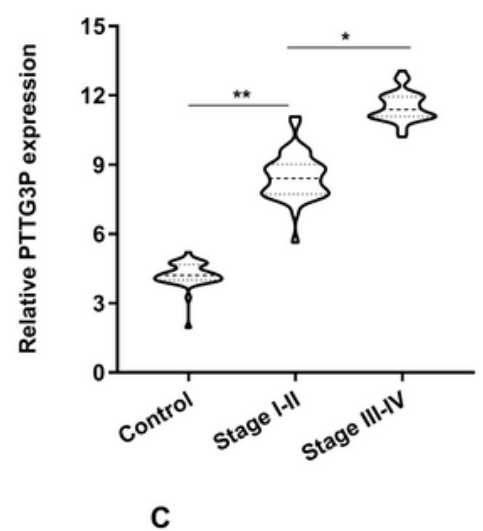

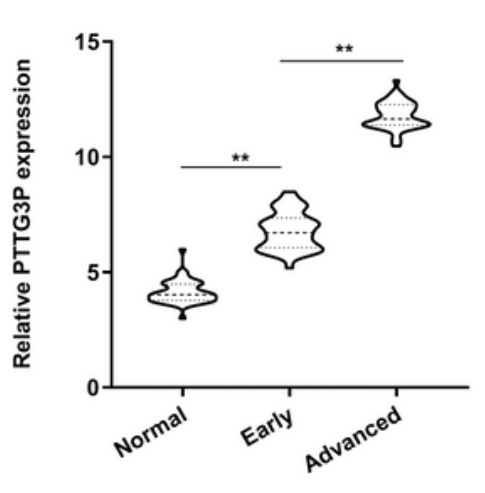

D
B

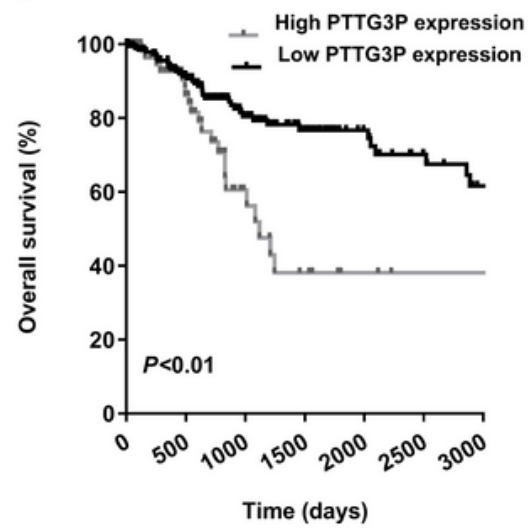

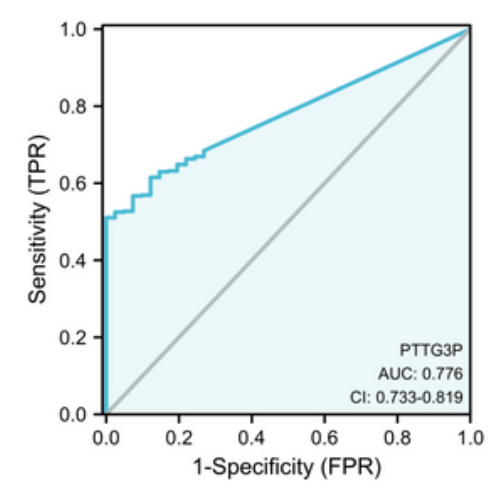

E

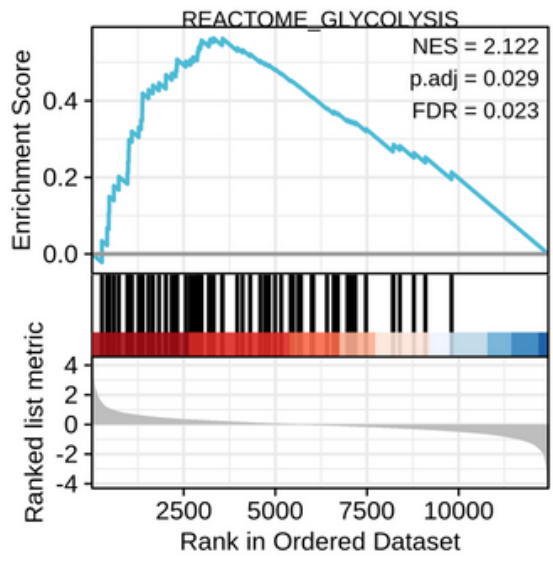

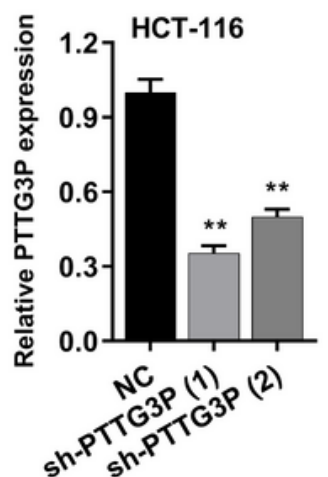

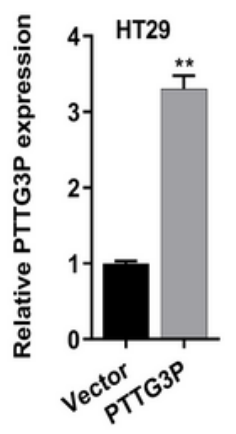

F

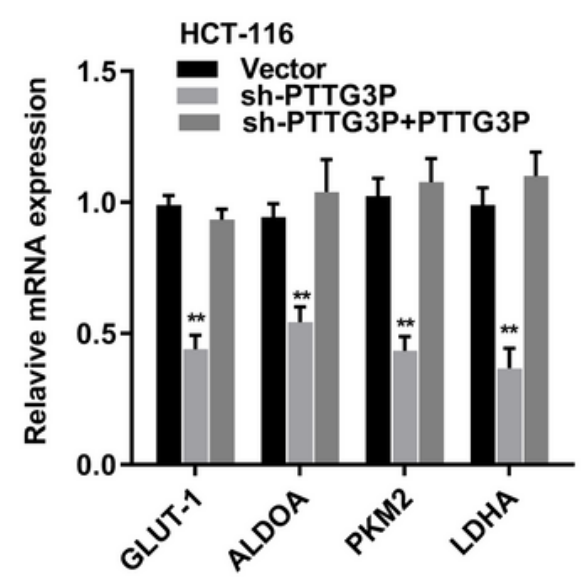

Figure 2

Overexpressed PTTG3P correlates with poor prognosis in CRC. (A) The expression of PTTG3P was checked in different clinical stages of CRC tissues (B) PTTG3P expression and survival predicted poor prognosis of OS in a cohort of 120 paired cases. (C) ROC curve of PTTG3P. (D) Short hairpin RNA 
(shRNA) targeting PTTG3P and PTTG3P overexpressed plasmids were transfected into HCT116 and HT29 cells. (E) GSEA plot showing that PTTG3P expression positively correlated with glycolysis-activated gene signatures (REACTOME GLYCOLYSIS). (F) Analysis of glycolic gene expression in PTTG3P knockdown or re-expressed PTTG3P. (G) qPCR analysis showed that PTTG3P was upregulated under low glucose culture conditions compared to normal glucose in a dose-dependent manner and time-dependent manner. (H) QPCR analysis showed that PTTG3P was increased after 2-DG treatment in dose-dependent manner and in time-dependent manner. ${ }^{*} \mathrm{P}<0.05, * * \mathrm{P}<0.01$, ${ }^{\star \star *} \mathrm{P}<0.001$

A
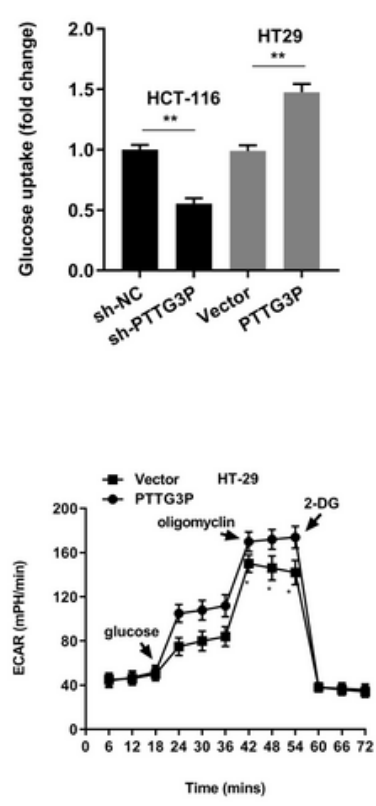

$\circ$

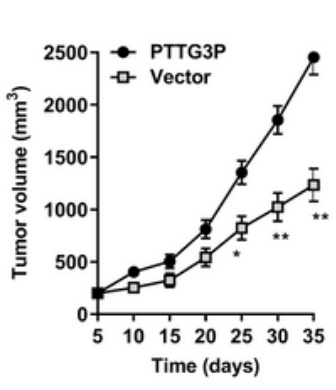

K

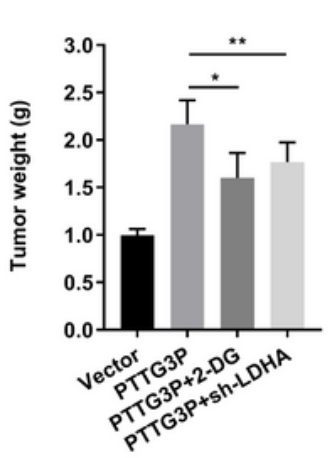

B

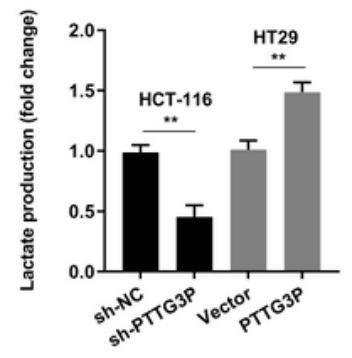

E

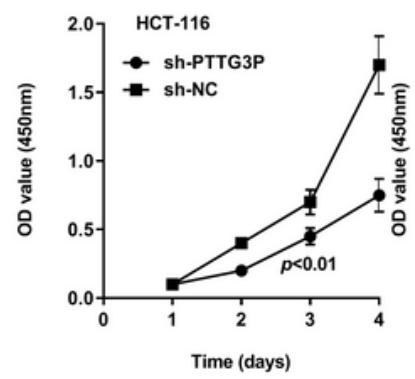

H

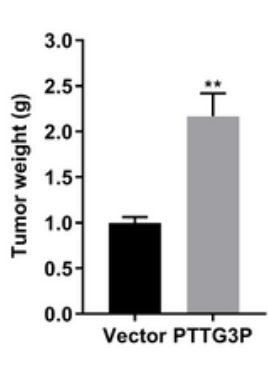

C
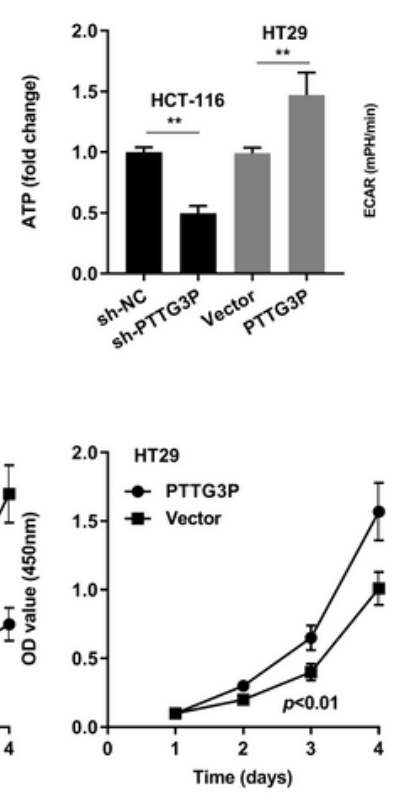

D

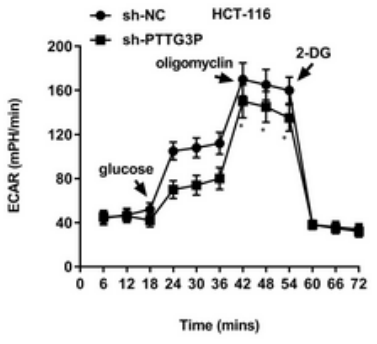

F

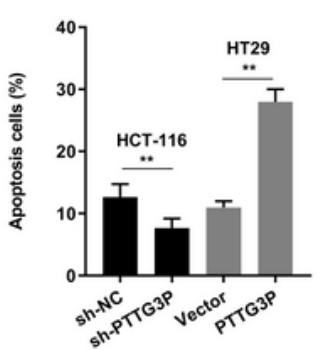

I

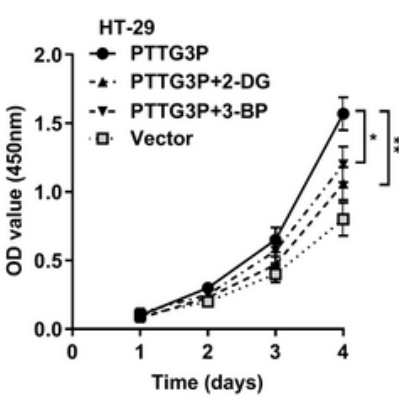

J

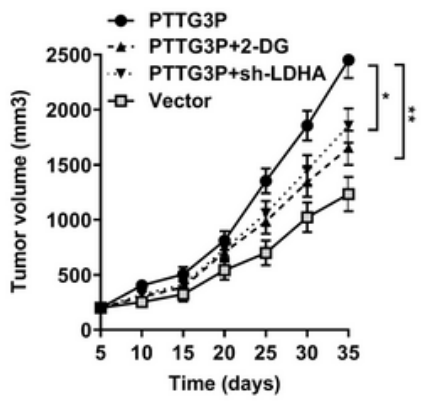

L

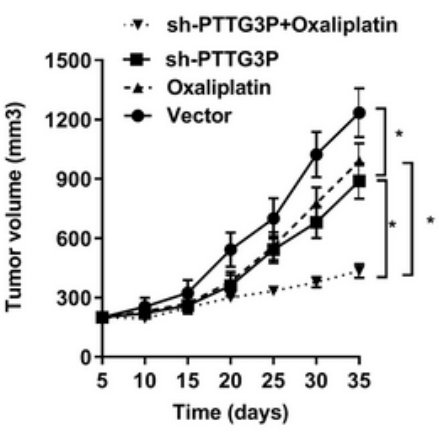

M

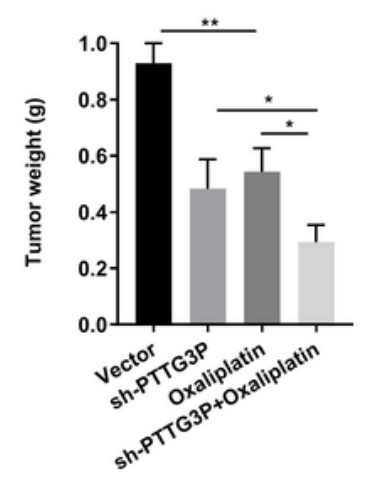




\section{Figure 3}

PTTG3P promotes the glycolysis and proliferation of CRC. (A) Glucose uptake analysis, (B) Lactate production analysis, (C) ATP analysis explored the glucose uptake, lactate production, and ATP accumulation level, with sh-PTTG3P or overexpressed PTTG3P in HCT116 or HT-29 cells. (D) Extracellular acidification rate (ECAR) analysis tested the glycolytic capacity in CRC cells with sh-PTTG3P or overexpressed PTTG3P in HCT116 or HT-29 cells. (E) CCK8 assay detected the proliferation of HCT116 and HT-29 cells transfected with sh-PTTG3P or overexpressed PTTG3P. (F) Flow cytometry assays revealed that PTTG3P affected cell apoptosis. $(\mathrm{G})$ Tumor volume and $(\mathrm{H})$ weight were measured in vivo when injected with overexpressed PTTG3P in HCT116 cells. (I) CCK8 assay detected the proliferation of HT-29 cells transfected with overexpressed PTTG3P and treated with 2.5mM 2-DG or $100 \mu \mathrm{M}$ 3-BP. (J) Xenograft tumors volume, $(\mathrm{K})$ Xenograft tumors weight were established, with injected with PTTG3P or PTTG3P plus sh-LDHA or PTTG3P treated with 2-DG $(1000 \mathrm{mg} / \mathrm{kg}$, injected into the abdominal cavity). Empty vector as indicated. (L) Tumor volume and (M) weight were measured in vivo when injected with sh-PTTG3P (20 nmol twice per week) and oxaliplatin treatment $₫ 5 \mathrm{mg} / \mathrm{kg}$ twice per week, injected into the abdominal cavity $₫$ transfected HCT116 cells. Data are presented as the mean \pm SD from three independent experiments. ${ }^{*} \mathrm{P}<0.05,{ }^{*} \mathrm{P}<0.01, * \star * \mathrm{P}<0.001$ 
A

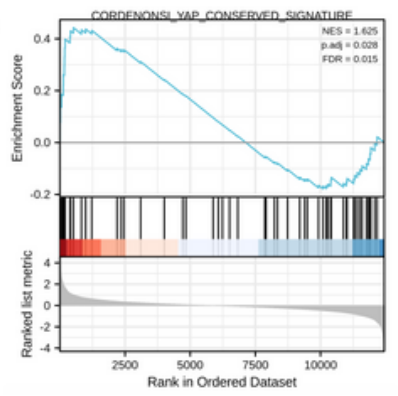

B

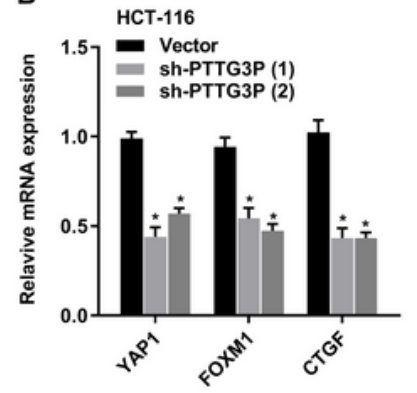

C

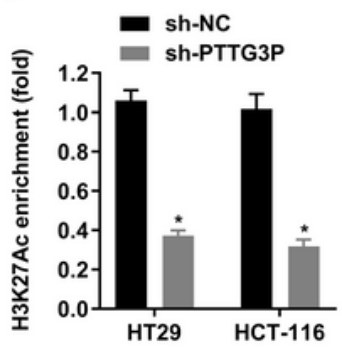

D
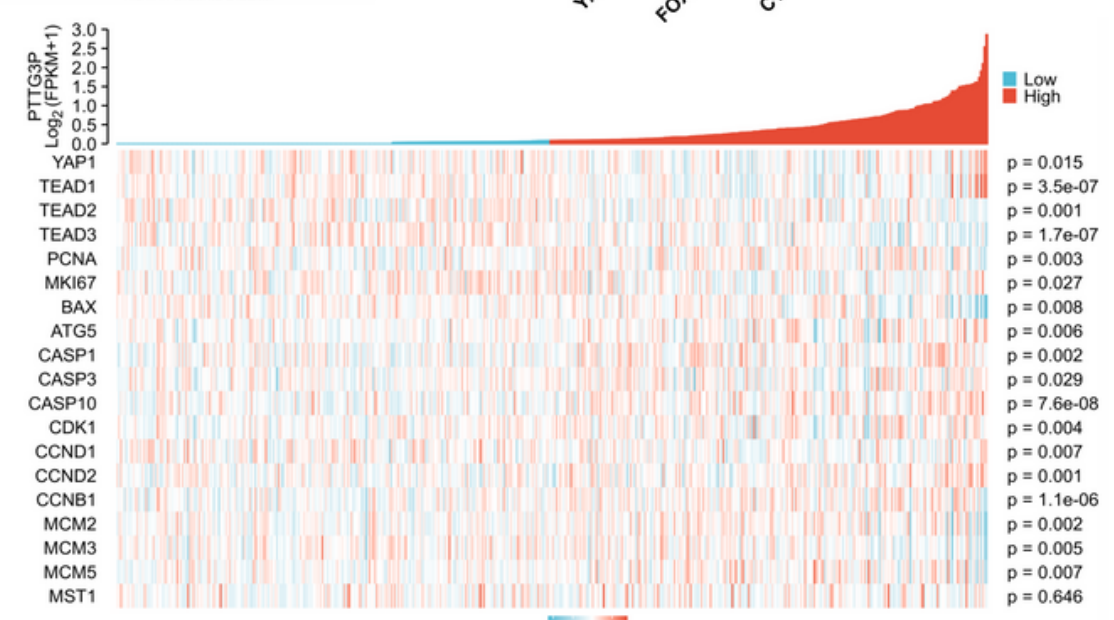

E
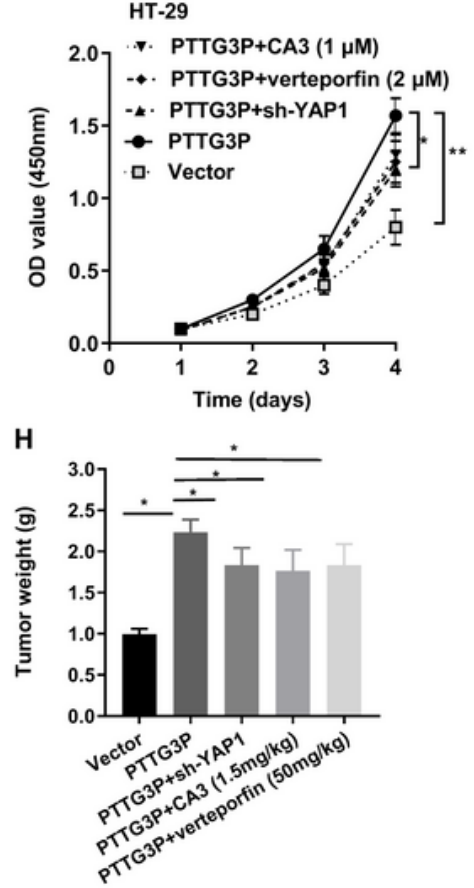

$\mathrm{F}^{\text {Z-score. }}$
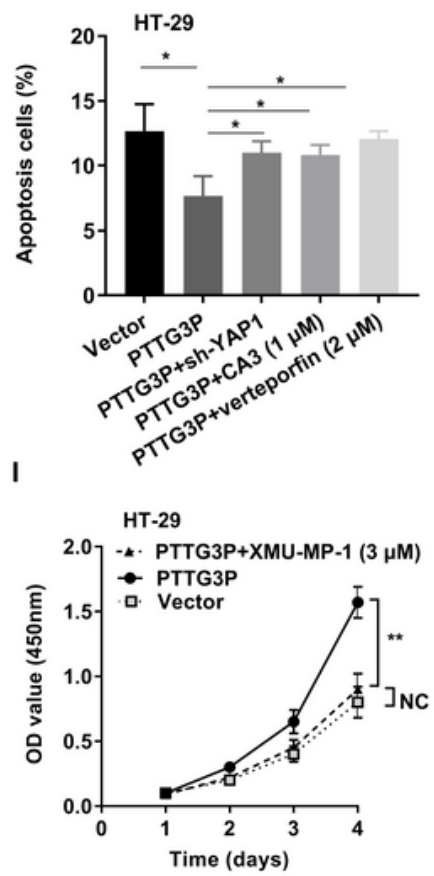

G

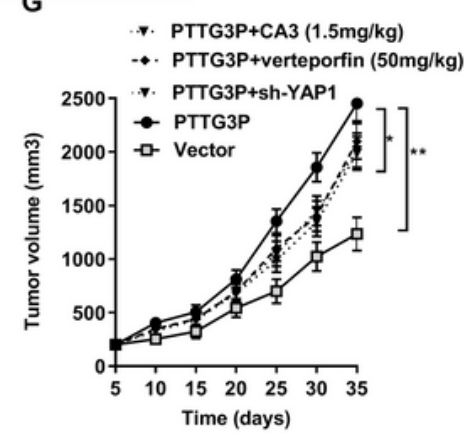

J

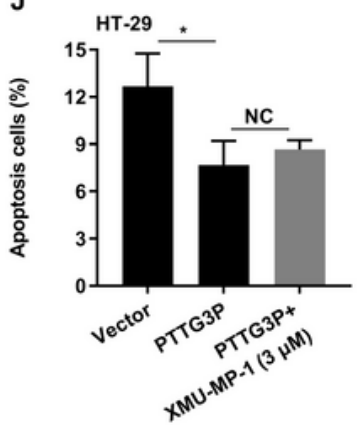

\section{Figure 4}

PTTG3P regulates Hippo signaling pathway in CRC (A) GSEA plot showing that PTTG3P expression positively correlated with YAP-activated gene signatures. (B) PTTG3P knockdown impaired the mRNA level of YAP1, FOXM1, and CTGF. (C) PTTG3P knockdown impaired the enrichment of H3K27Ac, H3K27me3 on the promoter of YAP1. (D) PTTG3P co-expression heat map, TCGA ( https://portal.gdc.cancer.gov/ ) COAD, level 3 HTSeq-FPKM. (E, F) The effect of HT-29 cells transfected 
with PTTG3P or PTTG3P+sh-YAP1 or PTTG3P+CA3 $(1 \mu \mathrm{M})$ or PTTG3P+verteporfin $(2 \mu \mathrm{M})$ on cell proliferation by CCK8 assay and apoptosis by flow cytometry assays. $(G, H)$ Xenograft tumor volume, Xenograft tumor weight were established, with injected with PTTG3P or PTTG3P+sh-YAP1 or PTTG3P+CA3 $(1.5 \mathrm{mg} / \mathrm{kg})$ or PTTG3P+verteporfin $(50 \mathrm{mg} / \mathrm{kg})$. (I) CCK8 assay detected the proliferation of HT-29 cells transfected with PTTG3P or PTTG3P plus XMU-MP-1 $(3 \mu \mathrm{M})(\mathrm{J})$ Flow cytometry assays revealed that PTTG3P plus XMU-MP-1 $(3 \mu \mathrm{M})$ could barely rescue cell apoptosis. Data are presented as the mean \pm SD from three independent experiments. ${ }^{*} P<0.05,{ }^{*} \mathrm{P}<0.01,{ }^{* *} \mathrm{P}<0.001$

A

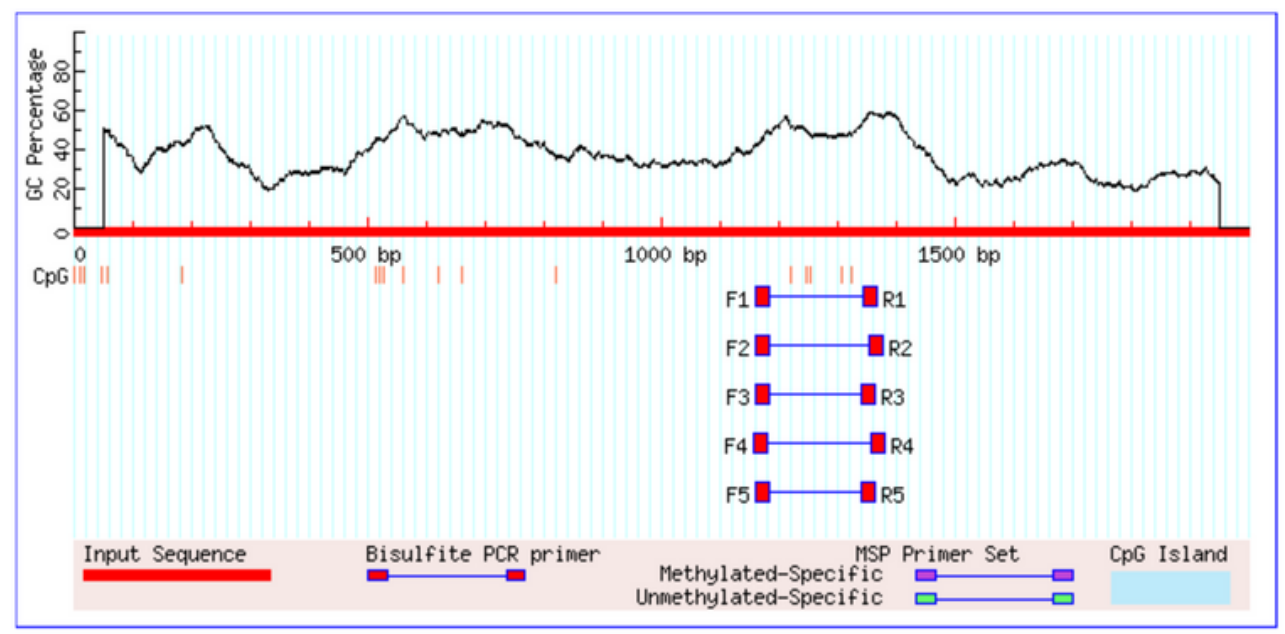

B

- CpG island region $\mathrm{CPG}$ site The heaviest CPG density island DNA sequence input

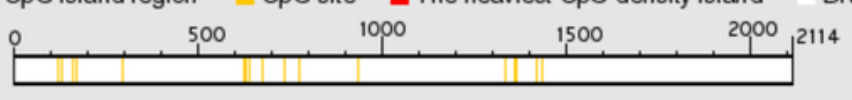

C

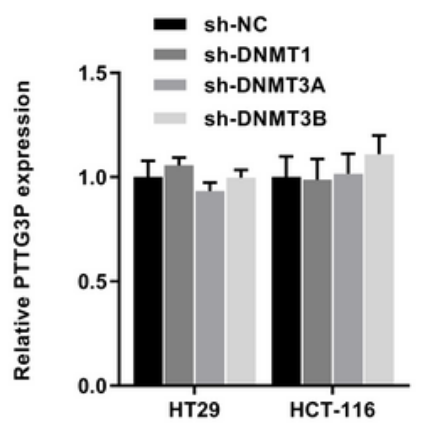

$\mathbf{F}$
D

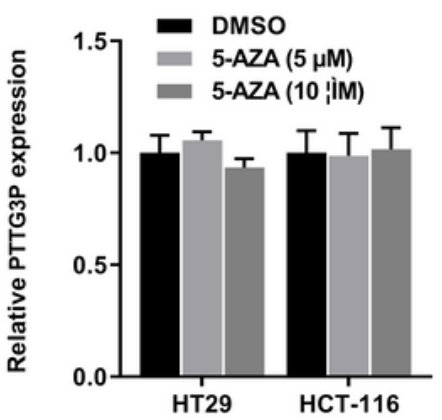

E

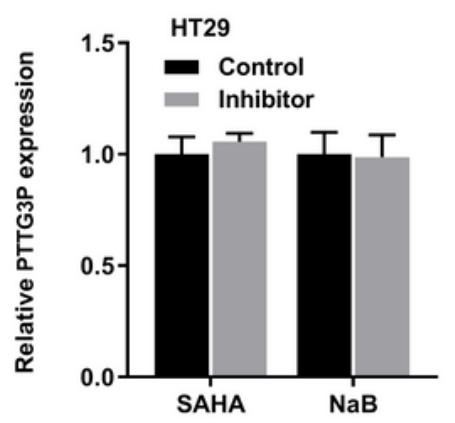

G
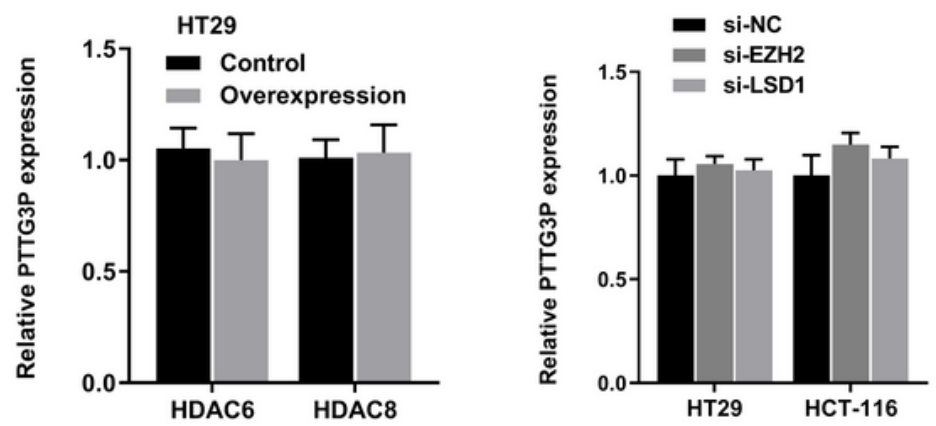


\section{Figure 5}

Methylation and acetylation do not affect the expression of PTTG3P. (A, B)Prediction of CpG islands in PTTG3P promoter region by analyzing the sequences of PTTG3P promoter through the MethPrimer online software (http://www.urogene.org/cgibin/methprimer/methprimer.cgi) and DBCAT (http://dbcat.cgm.ntu.edu.tw/) (C)The role of silencing DNMT1, DNMT3A, DNMT3B on the expression of LINC00301 in HT29 and HCT-116 cells. (D) The role of 5-AZA ( $5 \mu \mathrm{M}$ or $10 \mu \mathrm{M})$ treating for $72 \mathrm{hr}$ on the expression of PTTG3P in HT29 and HCT-116 cells. (E) The role of broad-spectrum HDAC inhibitors ( SAHA and $\mathrm{NaB}$ ) on the expression of PTTG3P in HT29 cells. (F) The role of overexpressed HDAC6 and HDAC8 on the expression of PTTG3P in HT29 cells. (G) The role of silencing EZH2 and LSD1 on the expression of PTTG3P in HT29 and HCT-116 cells. Data are presented as the mean \pm SD from three independent experiments. ${ }^{*} \mathrm{P}<0.05,{ }^{*} \mathrm{P}<0.01,{ }^{* \star *} \mathrm{P}<0.001$ 
A

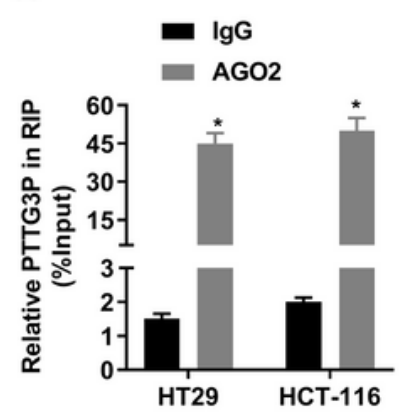

D

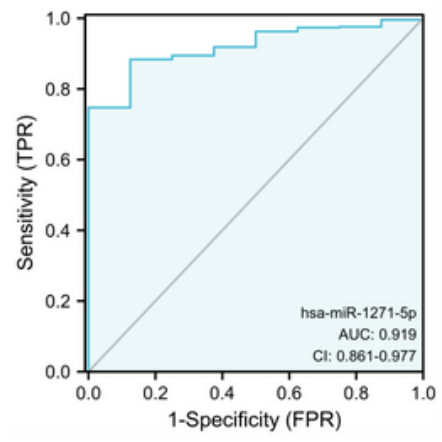

G

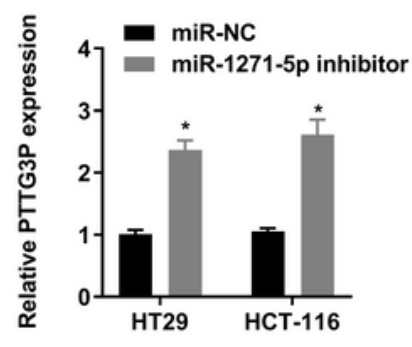

H

PTTG3P WT

hsa-miRNA-1271-5p

PTTG3P Mut
5' ...CUUAAAUCGT GCC AA...

| | | | | | |

3' ...GAACGAUCCA CGG UU...

5' ...CUUAAAUCCA CGG UU...
C

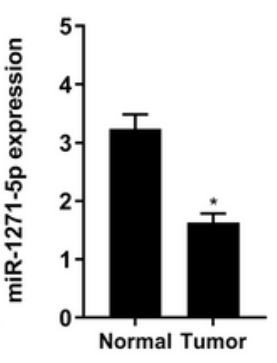

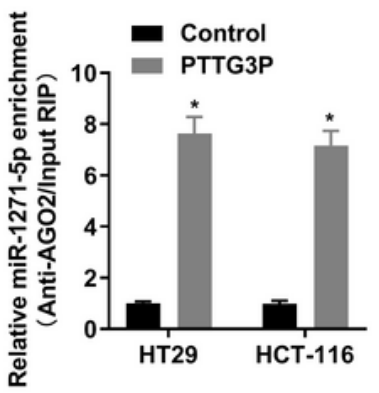

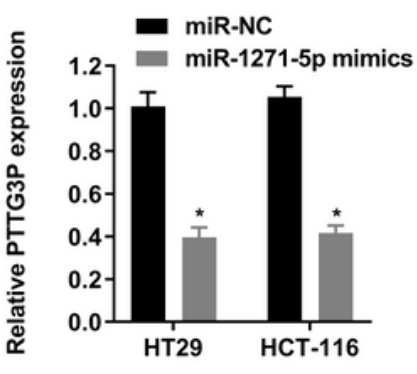

J

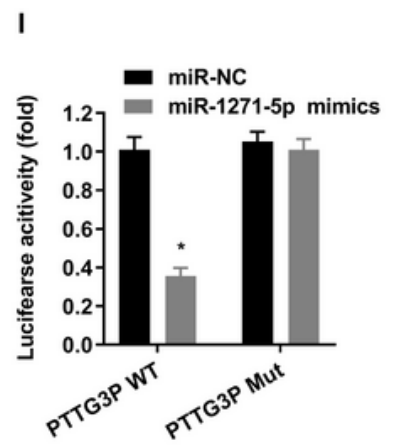

K
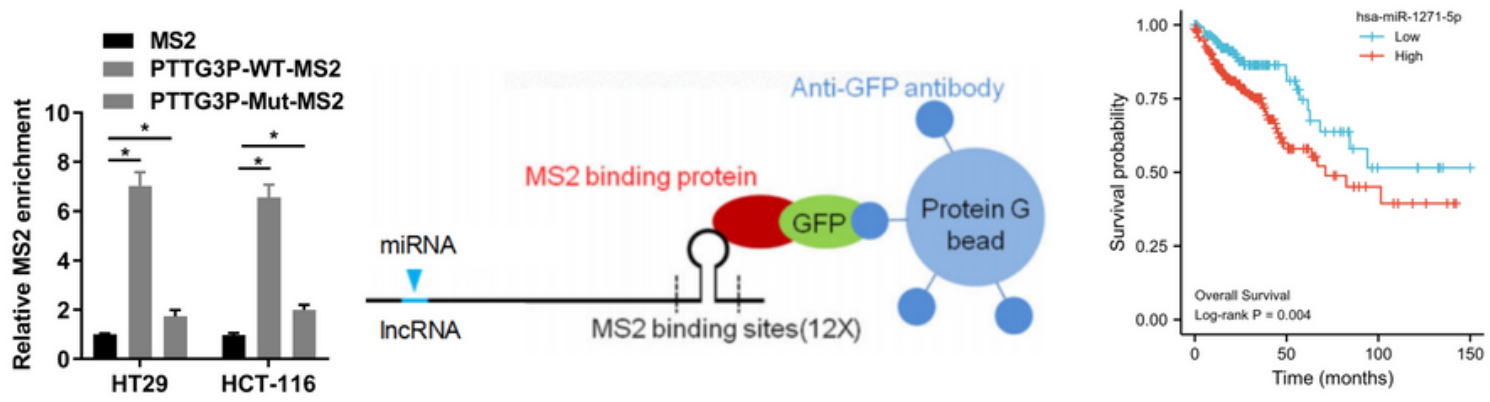

Figure 6

miR-1271-5p could interact with PTTG3P (A) Ago2-RIP assay and RT-PCR were performed in CRC cells to identify coprecipitated RNA. (B) Low miR-1271-5p expression was observed in the TCGA database of CRC $(n=465)(C)$ Low miR-1271-5p expression was observed in 120 paired tumor and paired adjacent nontumor tissues. (D) ROC curve analysis of the diagnostic value of miR-1271-5p. (E) Ago2-RIP assays showed the enrichment of PTTG3P in CRC cells transfected with miR-NC or miR-1271-5p. (F, G) 
Regulation of PTTG3P in CRC cells with miR-132-3p mimics or inhibitors. $(H)$ The predicted miR-1271-5p binding sites in the PTTG3P transcript. (I) Dual-luciferase assays implied that PTTG3P-WT instead of PTTG3P-Mut was targeted by miR-1271-5p. (J) MS2-RIP assay was used to further verify the direct interaction between miR-1271-5p and PTTG3P. A schematic outline of the MS2-RIP strategy was shown. (K) miR-1271-5p expression and survival curve of OS. ${ }^{*} P<0.05, * * P<0.01, * * * P<0.001$

A

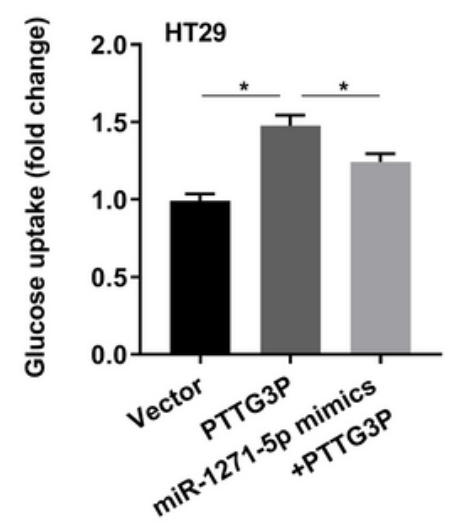

D

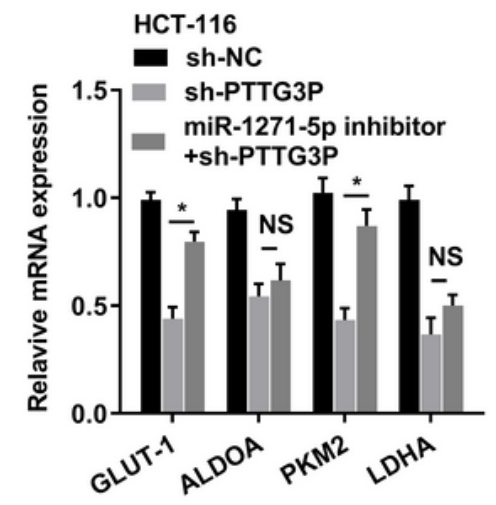

B

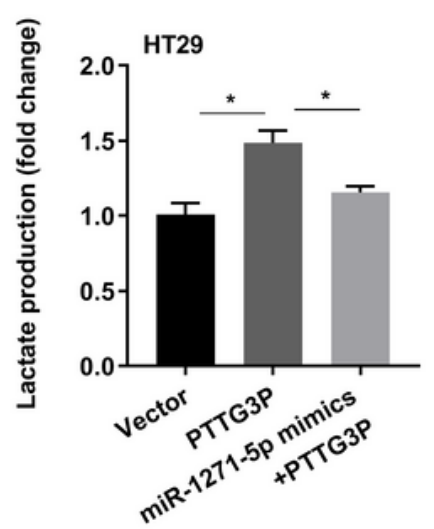

E

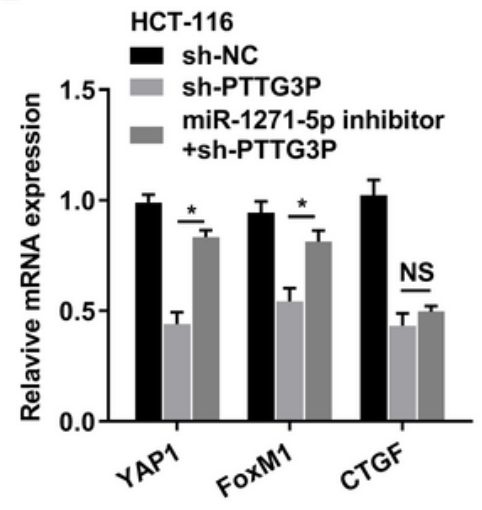

C

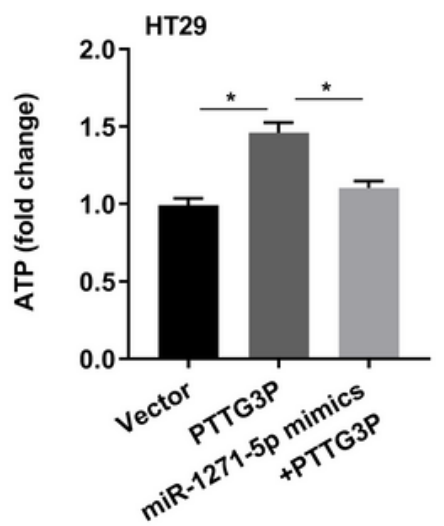

F $\square$ Vector

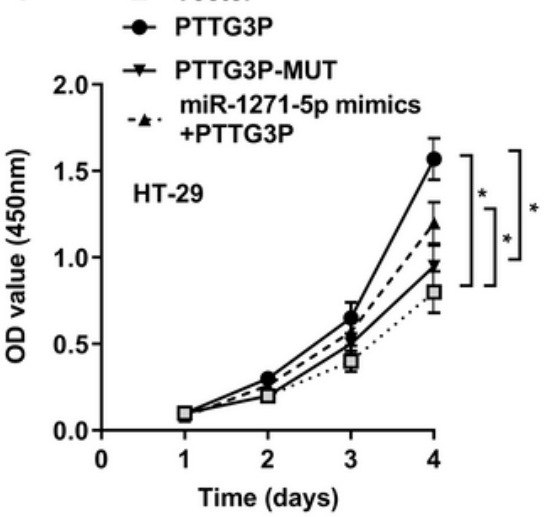

G

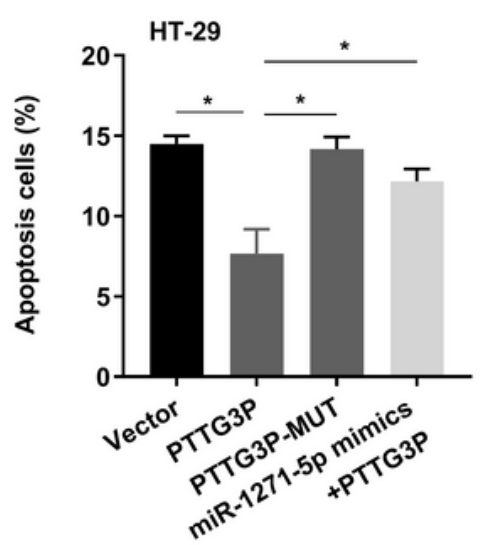

H

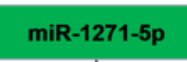

miR-1271-5p

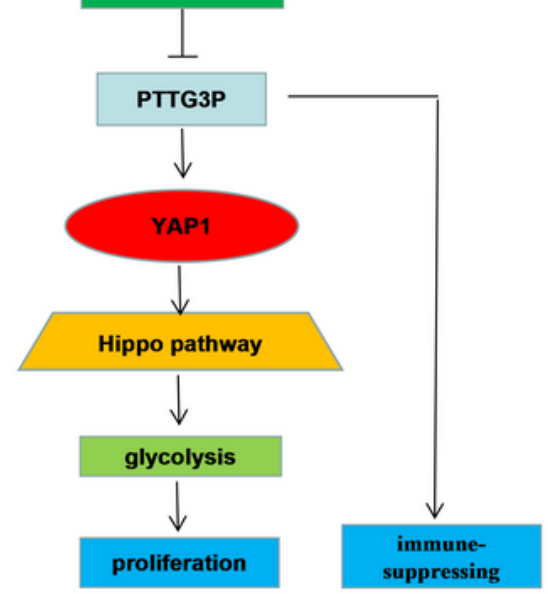

Figure 7 
miR-1271-5p could abrogate PTTG3P function (A-C) PTTG3P+miR-1271-5p mimics could rescue Glucose uptake analysis, Lactate production, ATP production in HT29 cells. (D, E) sh-PTTG3P+miR-1271-5p inhibitor could rescue the expression of glycolic genes, the expression of YAP1, FOXM1 and CTGF in HCT116 cells. (F-G) PTTG3P+miR-1271-5p mimics could rescue cell proliferation and apoptosis, but PTTG3PMUT could not increase cell proliferation or decrease apoptosis in HT29 cells. $(H)$ The expression of PTTG3P in HT-29 cells was measured after culturing under normoxia, hypoxia (1\% 02), or CoCl2 (100 $\mu \mathrm{M})$ for $24 \mathrm{~h}$ by qRT-PCR. (I) The expression of PTTG3P was evaluated by qRT-PCR in HT-29 cells after knockdown of HIF1A under normoxia condition. (J) The expression of PTTG3P was evaluated by qRTPCR in HT-29 cells after knockdown of HIF1A under hypoxia condition. (k) PTTG3P correlated with HIF1A. (L) The expression of miR-1271-5p in HT-29 cells could be inhibited by HIF1A. (M) Schematic representation of our study. ${ }^{\star} P<0.05,{ }^{\star *} \mathrm{P}<0.01$, ${ }^{\star \star *} \mathrm{P}<0.001$

\section{Supplementary Files}

This is a list of supplementary files associated with this preprint. Click to download.

- figureS1.tif

- figureS2Nomogram.tif

- figureS3.tif

- supplementarytable.doc 